\title{
Origin of Blazar Activity
}

\author{
M. M. Romanova \\ Space Research Institute of the Russian Academy of Sciences, Moscow, \\ Russia; and Department of Astronomy, Cornell University, Ithaca, NY \\ 14853-6801; romanova@astrosun.tn.cornell.edu
}

\begin{abstract}
.
Models of Blazars based on the propagation of finite discontinuities or fronts in the Poynting flux jet from the innermost regions of an accretion disk around a black hole are discussed. Such fronts may be responsible for short time-scale (from less than hours to days) flares in different wavebands from high frequency radioband to $\mathrm{TeV}$, with delay in low radio frequencies as a result of synchrotron self-absorption. The cases of magnetic fields of one and opposite polarities across the front are investigated. We find that annihilation of magnetic field in the front leads to higher energy spectrum of leptons and possibility of strong $\mathrm{TeV}$ flares. Electron-positron pairs form in most cases as a result of interaction between numerous synchrotron photons and SSC photons, and constitute the majority species, compared with the ions at subparsec scales. Frequent weak outbursts may be responsible for flickering core radiation in all wavebands, while the stronger outbursts may be observed as short time-scale flares.
\end{abstract}

\section{Introduction}

Blazars are characterized by fast variability in different wavebands from radio to gamma and in few cases by $\mathrm{TeV}$ radiation (Urry \& Padovani 1995; Punch 1992). Many of the objects reveal "superluminal" jets, which indicate that matter of the jet moves nearly toward us with relativistic speed (Blandford \& Rees 1978). The variability of the objects may be connected with outbursts of matter and energy from the nucleus and propagation of shocks along the collimated relativistic jet (Blandford \& Königl 1979; Marscher 1980). This model was further developed for investigation of different radio properties of radiogalaxies and quasars (Aller, Aller \& Hughes 1985; Hughes, Aller \& Aller 1985). The back extrapolated time of VLBI outbursts approximately coincides with strong optical, X-ray and gamma-ray flares (Kinman 1977; Belokon 1988; Krichbaum et al. 1995; Otterbein et al. 1998) which is in favor of this model. The idea of matter outbursts from centers of galaxies was first proposed by Ambartsumian (1958), and this has been confirmed by numerous observations.

The outbursted matter may be a normal electron-ion plasma, or it may consist mainly of electron-positron pairs. Prior to the Compton Observatory measurements, prediction of strong, collimated gamma-ray emission and elec- 
tron/positron cascades in AGN relativistic jets was made by the model of Lovelace, MacAuslan \& Burns (1979); Burns \& Lovelace (1982). More recently, a number of theoretical models have been developed to explain the observed gamma-ray emission of AGNs. In most of the models the gamma-ray radiation is ascribed to inverse Compton (IC) scattering of relativistic electrons and possibly positrons (Lorentz factors $\gamma \sim 10^{2}-10^{5}$ ) of a jet having relativistic bulk motion (Lorentz factor $\Gamma \sim 10$ ) with soft photons (energies $\sim 1-10^{2} \mathrm{eV}$ ). The soft photons can arise from the synchrotron emission of the relativistic electrons in the jet as in the synchrotron-self-Compton (SSC) models (Maraschi, Ghisellini \& Celotti 1992; Marscher \& Bloom 1992), or from the direct or scattered thermal radiation from an accretion disk (Dermer, Schlickeiser \& Mastichiadis 1992; Blandford 1993; Sikora, Begelman \& Rees 1994), or from a single cloud (Ghisellini \& Madau 1996). In a very different class of models, ultra high-energy protons (Lorentz factors $>10^{6}$ ) are postulated to cause a cascade, the product particles of which produce the observed radiation (Mannheim \& Biermann 1992; Protheroe \& Biermann 1997).

The idea of Poynting flux outbursts of energy to the jet is based on the fact that the central regions of the disk and a black hole may accumulate strong poloidal magnetic field of the order $B \sim\left(10^{3}-10^{4}\right) \mathrm{G}$. Rotation of this configuration leads to generation of the Poynting flux, which is a "permanent machine" for matter acceleration (Blandford \& Znajek 1977; Lovelace, Wang \& Sulkanen 1987; Livio, Ogilvio \& Pringle 1998). Recently, this idea was further developed and applied to gamma-ray Blazars by Romanova \& Lovelace (1997) (hereafter RL97), Colgate \& Li (1998) and by Levinson (1998). RL97 proposed that the main driving force for the observed superluminal jet components is a finite amplitude discontinuity in a Poynting flux jet. A rapid change in the Poynting jet outflow from a disk can result from implosive accretion in a disk with an ordered magnetic field (Lovelace, Romanova \& Newman 1994, hereafter LRN94). Propagation of newly expelled electromagnetic field and matter from the disk with higher velocity than the old jet can lead to the formation of a pair of shock waves as in the non-relativistic hydrodynamic flows in optical jet in protostellar systems (Raga et al. 1990). Particle acceleration in the front may result from the shocks and/or from annihilation and reconnection of oppositely directed magnetic fields in the front (Romanova \& Lovelace 1992, hereafter RL92; Lovelace, Newman \& Romanova 1997, hereafter LNR97). Here, we consider the different aspects of the flares of Blazars interpreted in terms of discontinuities in a Poynting flux jet. We analyze different theoretical and observational aspects connected with such outbursts.

\section{Magnetic Field in Jets}

Accreting matter in a disk around a black hole carries with it an ordered and a chaotic magnetic field. When the matter reaches the black hole, small magnetic loops reconnect and the field annihilates. The more ordered component of the field, which can have open field lines, can be dragged into the black hole while remaining connected to more distant plasma in the corona of the disk (Figure 1). Thus, the black hole may have a significant magnetic field passing through it supported by external currents in the disk and corona (Blandford \& Znajek 1977; 
Macdonald \& Thorne 1982). The magnetic field of the inner regions of the disk is likely to be comparable to the field in the black hole (Macdonald \& Thorne 1982; Livio et al. 1998). The area of the inner region of the disk is much larger than the area of the black hole, so that the magnetic flux and the Poynting energy outflow rate of the inner regions of the disk is larger than that of the black hole (Livio et al. 1998; Lovelace et al. 1987). The magnetic field near the inner edge of the disk is deduced to be of the order of $B \sim\left(10^{3}-10^{4}\right) \mathrm{G}$ (Lovelace 1976). Semi-empirical models of gamma-ray flares based on inverse Compton and/or SSC mechanisms, predict approximate values of the magnetic field in different Blazars in the regions of origin of the radiation (e.g., Sambruna, Maraschi \& Urry 1996; Sambruna et al. 1997). Back extrapolated to the inner disk using $B \sim 1 / r_{j}\left(r_{j}(z)\right.$ is the jet radius), gives a magnetic field $B=\left(10^{2}-10^{4}\right) \mathrm{G}$.

The rotating disk and black hole threaded by an ordered magnetic field generate Poynting flux outflows, or jets in which the energy density of the electromagnetic field is much larger than the matter energy density (see Lovelace, this volume).

\section{Poynting Flux Jets}

Poynting flux winds were first discussed by Goldreich and Julian (1968) for pulsars, and later Poynting flux jets were proposed to explain extragalactic jets by Lovelace (1976) and Blandford (1976). Solutions for Poynting flux outflows from a disk around a massive black hole were investigated by Lovelace et al. (1987).

A Poynting flux jet is self-collimated, with energy, momentum, and angular momentum transported mainly by the electromagnetic field (Lovelace et al. 1987). The collimation is due to the toroidal component of magnetic field. A steady Poynting flux jet is characterized in the lab frame by its asymptotic $\left(z>>r_{o}\right)$ magnetic field $B_{\phi}=-B_{0}\left[r_{0} / r_{j}(z)\right]$ and electric field $E_{r}=$ $-\left(v_{j} / c\right) B_{0}\left[r_{0} / r_{j}(z)\right]$ at the jet's edge with radius $r=r_{j}(z)$, where $r_{0}$ is the jet's radius at $z=0$. Also, $B_{0}$ is magnetic field at $z=0$, and $v_{j} \approx c$ is jet's axial velocity. The jet radius at $z=0$ is $r_{0} \sim(1-3) r_{g}=(2-6) G M / c^{2}$, where $r_{g}$ is the Schwarzschild radius, and $M$ is the black hole mass. The energy flux (luminosity) of the $+z$ jet is

$$
L_{j}=v B_{0}^{2} r_{0}^{2} / 8 \approx 3.0 \times 10^{43} \mathrm{erg} \mathrm{s}^{-1}\left(v_{j} / c\right) B_{3}^{2} M_{8}^{2},
$$

where $B_{3} \equiv B_{0} / 10^{3} \mathrm{G}, M_{8} \equiv M / 10^{8} M_{\odot}$, and $r_{0}=3 r_{g}$.

Matter accreting in the disk will be partially expelled to the jet by the Poynting flux "machine." An important quantity is the ratio of the magnetic energy to the matter kinetic energy at the base of the jet ,

$$
\mu \equiv\left(B_{0}^{2} / 8 \pi\right) /\left(\rho_{0} v_{j 0}^{2} / 2\right),
$$

where velocity at the base of the jet $v_{j 0}$ is assumed to be non-relativistic at this distance. The Poynting regime corresponds to $\mu>>1$. Note that in the Poynting flux regime, the magnetic field lines do not need to be inclined away from the $z$-axis in order for there to be energy outflow from the disk. This is in marked contrast with the hydromagnetic regime where the outflows require the 


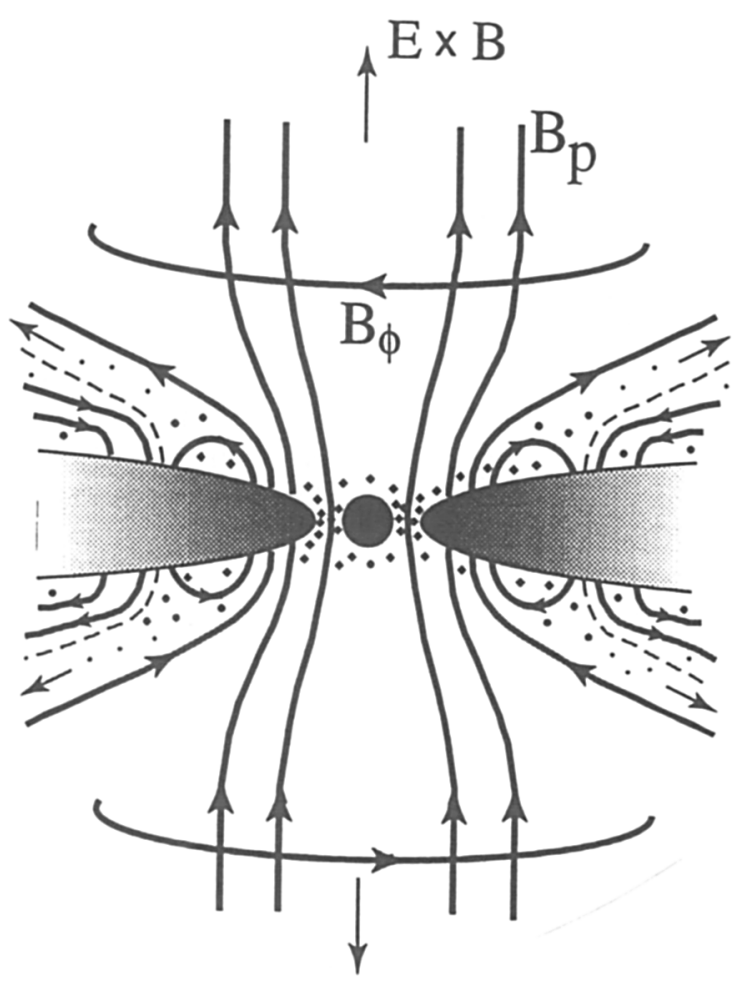

Figure 1. Sketch of magnetic field threading the inner region of an accretion disk around a black hole. A strong flux of electromagnetic energy (Poynting flux) forms as a result of twisting of the poloidal component of the magnetic field, and the fact that $\mathbf{E}=-\mathbf{v} \times \mathbf{B} / c$ in highly conducting plasma.

field to be inclined away from the $z$-axis by $>30^{\circ}$ (Blandford \& Payne 1982; Lovelace, Berk \& Contopoulos 1991; Romanova et al. 1997).

The matter flux carried by the jet is

$$
\dot{M}_{j}=\pi r_{0}^{2} \rho_{0} v_{j 0} \approx B_{0}^{2} r_{0}^{2} /(8 \mu c) \approx 5.2 \times 10^{-4} \mathrm{M}_{\odot} \mathrm{yr}^{-1} B_{3}^{2} M_{8}^{2} / \mu .
$$

At say $\mu=10$, the matter flux is $\dot{M}_{j}=5.2 \times 10^{-5} \mathrm{M}_{\odot} / \mathrm{yr}$, which is quite small compared with the disk accretion rate needed to fuel an AGN, $\dot{M}_{a c c r} \sim M_{\odot} / \mathrm{yr}$.

\subsection{Non-stationary Accretion and Formation of Fronts}

The electromagnetic energy outflow from the inner part of the accretion disk and from the black hole may be relatively steady if the disk accretion flow is steady. There may of course be small inhomogeneities connected with changes in the density and magnetic field of the accreting matter. However, if the disk is unstable, then the accretion rate increases and magnetic field also increases as a result of matter compression. 
Inhomogeneity of the magnetic field threading the disk can lead to a "global magnetic instability" of the accretion disk, which is connected with angular momentum outflow to Poynting flux jets or hydromagnetic outflows (LRN94; Lubow, Papaloizou \& Pringle 1994; LNR97). If the magnetic field is enhanced at some radius $r$ in the disk, then angular momentum will be the more efficiently lost from this region of the disk's surfaces to the jets. As a result, this disk matter will accrete faster and will accumulate matter in front of it as it moves radially inward. This will in turn amplify the magnetic field and further increase the loss of angular momentum to jets. Simulations of this process have shown that a strong wave-pulse of dense matter with strong magnetic field forms in the disk and propagates inward (LRN94). In case of a relatively high turbulent or " $\alpha$ " viscosity, a soliton-like wave forms (LRN94), while in case of a zero viscosity disk, a shock-like wave forms (LNR97). When this wave reaches the inner part of the disk, it generates the strong outburst of energy, angular momentum and matter to the jet. The pulse brings in a stronger magnetic field to the inner regions of the jet, which increases the Poynting flux. Thus, the new jet outflow will have a larger magnetic field, and can form a front propagating outward along the channel of the "old", weaker jet. Also, the new jet outflow may involve a reversal of polarity of the magnetic field (Romanova et al. 1998) so that the toroidal fields of the "new" and "old" jets are opposite (LNR97).

\subsection{Particle Acceleration in Propagating Front of Jet}

The plasma newly launched to the Poynting flux jet propagates along the old channel of the jet forming a front. The front is bounded by two shock waves - the first between the front and upstream matter and the second between the front and the downstream matter. Parameters of matter in the front are those in between the "old" and "new" jets. The difference in the energy fluxes between the "old" and "new" jets necessarily goes into the acceleration of particles inside the front (RL97). It is not possible from first principles to calculate the spectrum of accelerated particles because the acceleration mechanism is not known. Relativistic MHD shock acceleration may be important (Eilek \& Hughes 1990), but this is likely to accelerate mainly the ions if the plasma consists mainly of electrons and ions. From the other side, if the toroidal magnetic field reverses polarity across the front, then reconnection of magnetic field may be the dominant particle acceleration mechanism. Also, both particle acceleration mechanisms may be significantly different if the plasma consists mainly of electrons and positrons. Figure 2 shows sketch of possible configurations of the magnetic field in the front.

If accreting matter in the disk carries poloidal magnetic field of both polarities this can lead to reversals in the polarity of the toroidal magnetic field of the Poynting jet (see LNR97). This situation is sketched in Figure 2a. The case of a chaotic magnetic field (Figure $2 \mathrm{~b}$ ) may be always present in the plasma. Compression of plasma will lead to driven collisionless reconnection (Alfvén 1968; RL92).

RL97 proposed an empirical lepton spectrum motivated by observations where the energy of leptons is distributed as $f_{l} \sim \gamma^{-2}$ between energies $\gamma_{1}$, and $\gamma_{2}$ (in units of the rest mass energy), and is steeper $\sim \gamma^{-3}$ between energies $\gamma_{2}$ and $\gamma_{3}$. The lowest energy $\gamma_{1}$ was equal to that corresponding to synchrotron 
a

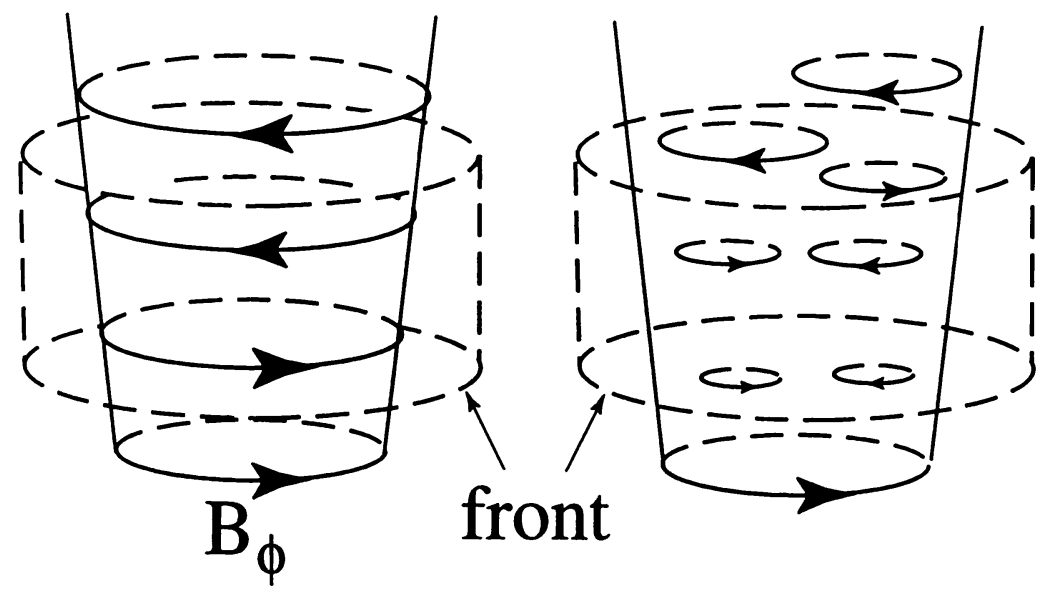

Figure 2. Sketches of possible configurations of magnetic field with opposite polarity, (a) an ordered field and (b) a chaotic field.

self-absorption. The average energy $\bar{\gamma}$ was calculated from the basic equations for the conservation of energy, mass and momentum. The energies $\gamma_{2}$, and $\gamma_{3}$ were derived from other considerations (see RL97). The actual spectrum $f_{l}$ may be somewhat different and will be determined by details of the acceleration process(es).

The basic equations for the evolution of the front follow from the conservation of mass, momentum, and energy,

$$
\begin{gathered}
\frac{\partial n_{i}^{\prime}}{\partial t^{\prime}}=-\frac{\partial\left(n_{i}^{\prime} v^{\prime}\right)}{\partial z^{\prime}}+\left(\frac{\partial n_{i}^{\prime}}{\partial t^{\prime}}\right)_{p a i r s}, \\
\frac{\partial T_{0 z}^{\prime}}{\partial t^{\prime}}=-\frac{\partial T_{z z}^{\prime}}{\partial z^{\prime}}+g r a v+r a d, \\
\frac{\partial T_{o o}^{\prime}}{\partial t^{\prime}}=-\frac{\partial T_{o z}^{\prime}}{\partial z^{\prime}}-s y n-S S C-C o m,
\end{gathered}
$$

and an equation for the magnetic flux. Here, $T_{\alpha \beta}^{\prime}$ is the stress energy tensor of the matter and the electromagnetic field, the primes denote quantities in the frame of the front, and "grav," "syn," etc denote different forces or energy fluxes discussed by RL97.

The equations were solved for different intrinsic parameters (RL97), but the example used below corresponds to a black hole mass $M=3 \times 10^{8} M_{\odot}$, magnetic field at the base of the jet $B_{0}=10^{3} \mathrm{G}$, initial ion/lepton ratio $f_{l i}=1$, magnetic/particle energy at the base of the jet $\mu=15$, viewing angle $\theta=$ $0.2 \mathrm{rad}$, the luminosity of background photons $L_{p h}=10^{46} \mathrm{erg} \mathrm{s}^{-1}$, their energy $\epsilon_{p h}=10 \mathrm{eV}$, and radius of their distribution $R_{p h} \approx 3 \times 10^{17} \mathrm{~cm}$. The density and magnetic field ratios between the "old" and "new" matter are $n_{1} / n_{2} \approx 0.4$ and 
$B_{1} / B_{2} \approx 0.4$. The velocities correspond to a bulk Lorentz factors $\Gamma_{1}=8$ and $\Gamma_{2}=18$ (where $\Gamma=\left[1-\left(v_{j} / c\right)^{2}\right]^{-1 / 2}$ ). The cases of a single and reverse polarity of the magnetic field across the front were investigated and compared. After expulsion of new matter, the front accelerates up to $\Gamma \approx 12$, so that the Doppler boost factor is $\delta=1 / \Gamma\left[1-\left(v_{j} / c\right) \cos \theta\right] \approx 4$. Leptons are accelerated in the front from $\gamma=1$ to $\gamma_{1} \approx 10^{2}, \gamma_{2} \approx 10^{3}-5 \times 10^{3}$, and $\gamma_{3} \approx\left(6 \times 10^{3}-2 \times 10^{4}\right)$. In the case of reversal polarity leptons are accelerated up to higher energies: $\gamma_{2} \approx 10^{4}-10^{5}$, and $\gamma_{3} \approx 10^{5}-10^{6}$. These maximum values of $\gamma$ depend on the duration of expulsion of "new" matter with opposite polarity.

\subsection{Energy Release Due to Magnetic Field Reconnection}

Reconnection of the magnetic field may occur along the jet, in particular, in the front, where matter is compressed. The magnetic field at large distances is $B_{\phi}=-B_{0}\left(r_{0} / r_{j}\right)$. The magnetic energy-density at some distance $z$ along the jet is $B_{\phi}^{2} / 8 \pi$ so that the total magnetic energy in the front is $E_{m}=\left(B_{\phi}^{2} / 8 \pi\right)\left(2 \pi r^{3}\right)$, where we supposed that the region is a cylinder with a length equal to its diameter. If the magnetic field reverses polarity across the front, then this magnetic energy may be released entirely in the form of accelerated particles during an Alfvén time $t_{A}=2 r_{j} / v_{A}$, where the Alfvén velocity $v_{A}=c /\left(1+4 \pi \rho c^{2} / B_{\phi}^{2}\right)^{1 \gamma_{2}} \sim$ $c$. Thus, the "luminosity" owing to the reconnection is

$$
L_{\text {recon }} \sim E_{m} / t_{A}=B_{0}^{2} r_{0}^{2} c / 8 \sim 3.0 \times 10^{43} \mathrm{erg} \mathrm{s}^{-1} B_{3}^{2} M_{8}^{2} .
$$

The spectrum of leptons resulting from collisionless driven reconnection is a power law $\gamma^{-2}$ for electron-ion plasma, and $\gamma^{-1.5}$ for electron-positron plasma (RL92). In our analysis of the time evolution of the front in a Poynting flux jet, we took into account the annihilation of magnetic field in the case where the field reverses polarity across the front. We found larger particle energies in the case where the field reverses polarity.

\subsection{Pair Creation}

The particle content of the jets is not known (Krolik, this volume). In our model, the density of synchrotron photons inside the front is typically much larger $\left(10^{4}-10^{5}\right.$ times) than the density of the background photons (see also RL97). Interaction of the electrons with these photons produces a high density of high-energy SSC photons. Analysis of different possible mechanisms of pair creation leads to the conclusion that interaction of SSC photons with synchrotron photons is the most important process. A pair forms when $\epsilon_{s y n} \epsilon_{s s c}>\left(m_{e} c^{2}\right)^{2}$, where $\epsilon_{s y n}$ and $\epsilon_{s s c}$ are energies of synchrotron and SSC photons. For rough estimates, we can write $\epsilon_{s y n}=(3 / 2) \gamma^{2} \hbar \omega_{o}^{\prime}$, where $\omega_{o}^{\prime}=e\left|B^{\prime}\right| /\left(m_{e} c\right)$ is the cyclotron frequency in the front frame, $\epsilon_{s s c}=\gamma^{2} \epsilon_{s y n}$, and $\left|B^{\prime}\right|$ is the magnetic field strength in the front frame. An approximate condition for pair production is

$$
\gamma \geq \gamma_{\text {pair }} \equiv\left(m c^{2} / \hbar \omega_{o}^{\prime}\right)^{1 / 3} \approx 3.5 \times 10^{3}\left(\left|B_{3}^{\prime}\right|\right)^{-1 / 3} .
$$

Electron-positron recombination is negligible for the conditions considered. We observed that the pairs form at a variety of parameters of the model. In a typical case, the total number of pairs in the front $N_{l}$ grows proportionally to the total 

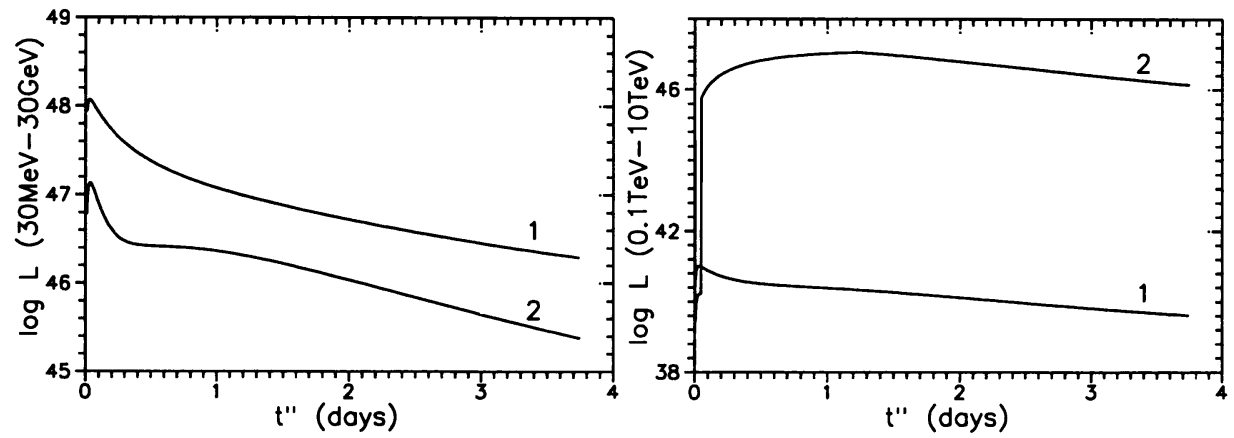

Figure 3. Luminosity of EGRET flares (left panel) and $\mathrm{TeV}$ flares (right panel) in erg/s for cases of (1) a magnetic field of one polarity, and (2) a field which reverses polarity across the front.

number of ions $N_{i}$ accumulated by the front. Thus, their ratio $f_{l i} \equiv N_{l} / N_{i}$ is almost constant during the front propagation and constitutes $f_{l i} \approx 10$ for $\mu=15$, and $f_{l i} \approx 100$ for $\mu=100$. The pair creation does not appreciably alter the energy distribution of leptons because the internal energy per particle is proportional to $\mu$ and the number of leptons is approximately proportional to $\mu$. In RL97, pairs did not form, because at similar initial parameters, they took initial value $f_{l i}=5$ at the base of the jet. Thus, leptons had smaller initial energy per particle and the condition $\gamma>\gamma_{\text {pair }}$ was not satisfied. Summarizing, we conclude that pairs form in many cases, but only in case of very low matter energy compared to magnetic energy, $\mu>>1$, their number may be significant compared to the number of ions.

In case of polarity reversals of the $B_{\phi}$ field (Figure 2), the magnetic field gradually annihilates and becomes weaker in the front frame. The pairs form in the beginning of the front propagation $\left(z<10^{16} M_{8} \mathrm{~cm}\right)$, when the magnetic field is strong enough to produce significant population of synchrotron and SSC photons. Later, the rate of pair creation decreases, and $f_{l i}$ decreases. The fact, that in case of polarity reversals, the internal energy of the front is larger (due to reconnection) while the number of leptons is smaller (due to weaker pair creation) leads to higher energies per lepton $\gamma$ and the entire spectrum is harder.

\subsection{Radiation from the Front}

Accelerated leptons interact with background and synchrotron photons. Each of three processes, synchrotron, IC, and SSC, give radiation in a broad bands of energies. They cover the whole range of energies from $10^{-3} \mathrm{eV}$ (radio with $\lambda \approx$ $1 \mathrm{~mm}$ ) up to $\sim 100 \mathrm{GeV}$ in case of single polarity and up to $(1-10) \mathrm{TeV}$ in case of polarity reversals. Thus, the Poynting flux outburst generates a simultaneous flare in all wavebands from high frequency radio up to high energy radiation. Here, we calculated the luminosity of flares in EGRET band $(30 \mathrm{MeV}-30 \mathrm{GeV})$ and in $\mathrm{TeV}$ band for energies $(0.1-10) \mathrm{TeV}$ (see Figure 3). The cases of one polarity (1) and two polarities (2) of the toroidal magnetic field are shown. One can see that the gamma-ray luminosity is higher in the case of a single polarity, whereas the $\mathrm{TeV}$ luminosity is much higher in the case of reversals of 
polarity of the field across the front. In case of one polarity, radiation in both energy bands is determined by SSC processes, while in case of polarity reversal, both gamma and $\mathrm{TeV}$ radiation are determined mainly by the IC processes (because the magnetic field becomes too weak to produce significant synchrotron and correspondingly SSC radiation). The fairly strong $\mathrm{TeV}$ flare in case of polarity reversals appears as a result of higher energy of leptons in the front compared with the case of a single polarity. The flare gradually decreases because the density of background photons decreases. However, the strong luminosity flare may appear later in the case of interaction with a Broad Line Region cloud (Ghisellini \& Madau 1996). The flares in X-ray and optical bands are smaller, than in the EGRET band. The detailed analysis of the spectrum will be considered in the future.

In the example shown (Figure 3) it was suggested that the "new" matter was outbursted from the disk during $\sim 100$ periods of rotation of the inner radius of the disk, $\Delta t=100\left(2 \pi r_{0} / v_{K 0}\right)$, which corresponds to $\Delta t^{\prime \prime} \approx 4$ days in the observer's frame $\left(d t^{\prime \prime}=d t(\Gamma / \delta)=d t\left[1-\left(v_{j} / c\right) \cos \theta\right]\right)$. If the source of new matter ceases earlier (see also Levinson 1998), then the flare may be much shorter, of the order of the dynamical time-scale at the inner edge of the disk, $\Delta t_{d y n}^{\prime \prime}=t_{d y n}(\Gamma / \delta) \approx 0.8(\Gamma / \delta) M_{8} \mathrm{hr} \approx 2.4 M_{8} \mathrm{hr}$. Thus, the flares shown at Figure 3, may be much shorter. The discussed model presents a definite answer regarding the amplitude of the flares, but their duration is somewhat uncertain and is determined by the processes in the inner regions of the disk. We expect that the inner regions of the disk may frequently be unstable owing to angular momentum outflow to the jet. This is an analogue of the "global magnetic instability" (LRN94; LNR97), but on smaller scales. This will lead to frequent outbursts of matter to the jet with a characteristic time, which may be as short as a few dynamical time-scales. One can expect numerous hour time-scale flares with less frequent, more powerful, day time-scale flares determined by stronger instabilities in the disk.

\section{Comparison with Observations}

In Blazars we are looking down the jet and this gives information not visible in other AGNs (Sambruna, these Proceedings). But how deep can one observe the inner jet? The interaction of gamma-ray photons with few $\mathrm{KeV}$ background radiation leads to electron-positron pair production (e.g., Burns \& Lovelace 1982; Blandford \& Levinson 1995). The corresponding $\gamma$-sphere has a radius (Takahara 1997): $r_{\gamma}>1.0 \times 10^{-5}\left(\epsilon_{\mathrm{ob}} / \mathrm{GeV}\right) L_{46} \delta_{10}^{-5} \mathrm{pc}$, where $\epsilon_{\mathrm{ob}}$ is the energy of the observed photons (in $\mathrm{GeV}$ ), and $L_{46}$ is the total luminosity of X-ray photons. At small background luminosities, and, specifically, at small boost factors $\delta$, the value $r_{\gamma}$ may be very close to the radius of the black hole. On the other hand, even if $r_{\gamma}>r_{g}$, then near the black hole, the X-ray radiation is expected to be anisotropic and come from the accretion disk. Thus, interaction of gamma-ray photons with background radiation may occur only at the "walls" of the jet, which may be opaque to penetration of X-ray photons (Illarionov \& Krolik 1996; Thompson 1997).

The fastest flares reported so far last a few hours as in the case of Mrk 421 (Gaidos et al. 1996), down to less than an hour in Mrk 501 (Aharonian et al. 
1999). In PKS 1622-297 the duration of the flare is a few days, but the rise time of the flare is only a few hours (Mattox et al. 1997). This indicates that the size of the emitting region is very small (unless the Doppler boost factor $\delta$ is not extremely large).

The Poynting flux outbursts discussed here lead to quite small variability timescales, from less than an hour up to possibly a few days. The short flares are much more probable. The frequent small-scale flares may explain the intraday variability in different wavebands from optics (and possibly high-frequency radio) up to $\mathrm{GeV}$ and $\mathrm{TeV}$ radiation.

What is the origin of the long-time flares, which may last a few weeks or even months? The long flares may be explained for example by superposition of smaller-scale flares which may occur frequently (Magdziarz, Moderski \& Madejski 1997; Chiaberge \& Ghisellini 1998). The overlapping of light-curves of small flares may determine the non-thermal continuum radiation in different wavebands from radio to gamma with flickering determined by the separate flares. The low-energy photons have a much longer cooling time (e.g. Atoyan \& Aharonian 1997), so that the flickering of the low-energy radiation may be smoothed significantly (Chiaberge \& Ghisellini 1998). However, we still cannot exclude the possibility that intraday $(\mathrm{mm})$ radio variability (e.g. Wagner \& Witzel 1995) of some radioquasars may be also determined by the expulsion of shocks from the region near the black hole.

The Poynting flux outburst model does not exclude the possibility of formation of longer flares at larger distances from the black hole. For example, the $\mathrm{TeV}$ flare in Mrk 501, may be explained by the injection of high-energy particles with $\gamma_{\max } \sim 10^{5}-10^{6}$ with luminosity $L_{j} \sim 10^{41} \mathrm{erg} \mathrm{s}^{-1}$ to a region with the size $R \sim 10^{16} \mathrm{~cm}$ (Maschidiadis \& Kirk 1997, see also Sambruna et al. 1998). These particles may originate in a shock wave or a reconnection event. This luminosity constitutes only one percent of magnetic field annihilation luminosity (eq. 7).

Correlated multiwavelength observations show near simultaneous outbursts in different wavebands from the optical to gamma-ray (e.g., Bloom et al. 1997), which support the Poynting flux model. Recent observations show clear correlation between $\mathrm{X}$-ray and $\mathrm{TeV}$ fluxs in $\mathrm{TeV}$ Blazars (e.g., Pian et al. 1998), which is a strong argument in favor of $\mathrm{SSC}$ mechanism of $\mathrm{TeV}$ radiation in these sources. The TeV flares found in our model are determined by IC radiation, and hence cannot be applied to these particular sources. In these objects additional acceleration of leptons may occur in shock waves or by $B$ field reconnection.

\section{Conclusions}

The main conclusions from the investigated models are:

1. The rapidly rotating inner region of an accretion disk threaded by magnetic field $\left(\sim 10^{3}-10^{4} \mathrm{G}\right)$ as well as the rapidly rotating black hole can generate field dominated or Poynting flux jets.

2. Accretion disk instabilities, specifically the "global magnetic instability" of the disk, can bring matter and magnetic flux rapidly to the inner region of the disk and thereby generate strong outbursts of energy to the jet. 
3. In the region between the "old" and "new" matter of the jet, a "front" forms, where particles are efficiently accelerated owing to shock waves and/or enhanced reconnection/annihilation of the magnetic field.

4. Flares in all frequency bands from IR (or $\mathrm{mm}$ ) to $\mathrm{GeV}$ (and in some cases $\mathrm{TeV}$ ) are expected to appear approximately simultaneously. In the case where the toroidal magnetic field reverses polarity across the front, strong $\mathrm{TeV}$ flares may occur.

5. The millimeter radio flux may have a short delay (hours) compared with other wavebands as a result of self-absorption. However, in some cases (where the magnetic field at the base of the jet is less than $\sim 10^{3} \mathrm{G}$ ) it may appear simultaneously with other wavebands. There is an even longer delay for lower frequency radio emission.

6 . Frequent weak outbursts to the jet may overlap and determine the nonthermal continuum radiation from radio to gamma-ray band. They may be observed as intraday variability in different wavebands.

7. The strongest outbursts may appear as short time-scale flares with duration from less than an hour up to a few days, depending on accretion processes in the disk and the radiation rate of the leptons.

8. Reconnection of magnetic field leads to locally accelerated leptons and the possibility of $\mathrm{TeV}$ flares. Small-scale reconnection events may determine the small-scale variability at large distances from the black hole with times less than $r_{j}(z) / c$, where $r_{j}$ is the radius of the jet.

Acknowledgments. The author thanks IAU for partial support, and the Local Organizing Committee for warm hospitality. This work was made possible in part by Grant No. RP1-173 of the U.S. Civilian R\&D Foundation for the Independent States of the Former Soviet Union.

\section{References}

Aharonian, F.A., et al. 1999, A \& A, 342, 69

Alfvén, H. 1968, JGR, 73, 4379

Aller, H.D., Aller, M.F., \& Hughes, P.A. 1985, ApJ, 298, 296

Ambartsumian, V.A. 1958, La structure et l'evolution de l'univers, Solvey Conference, p. 241, Bruxelles, ed. by R. Stoops

Atoyan, A.M., \& Aharonian, F.A. 1997, ApJ, 490, L149

Begelman, M.C., Blandford, R.D., \& Rees, M.J. 1984, Rev. Mod. Phys., 56, 255

Belokon, E.T. 1988, Astrophysics, 27, 588

Blandford, R.D. 1976, MNRAS, 176, 465

Blandford, R.D. 1993, in Proc. Compton Symp. 1992, ed. M. Friedlander, N. Gehrel, \& D.J. Macomb, New York: AIP, 553

Blandford, R.D. \& Königl, A. 1979, ApJ, 232, 34

Blandford, R.D., \& Levinson, A. 1995, ApJ, 441, 79

Blandford, R.D., \& Payne, D.G. 1982, MNRAS, 199, 883 
Blandford, R.D., \& Rees, M. 1978, in Pittsburgh Conference on BL Lac Objects, ed. A.M. Wolfe, Univ. Pittsburgh Press, 1978, p.328

Blandford, R.D., \& Znajek, R.L. 1977, MNRAS, 179, 433

Bloom, S.D., et al. 1997, ApJ, 490, L145

Burns, M.L., \& Lovelace, R.V.E. 1982, ApJ, 262, 87

Chiaberge, M., \& Ghisellini, G. 1998, MNRAS, astro-ph/9810263

Colgate, S.A. \& Li, H. 1997, in Relativistic Jets in AGNs, ed. M. Ostrowski, M. Sikora, G. Madejski and M. Begelman, Poland, Kraków, p.170

Dermer, C.D., Schlickeiser, R., \& Mastichiadis, A. 1992, A\&A, 256, L27

Eilek, J.A., \& Hughes, P.E., in Astrophysical Jets, ed. P.E. Hughes, Cambrudge University Press, Cambridge, p. 428

Gaidos, J.A. et al. 1996, Nature, 383, 319

Ghisellini, G., \& Madau, P. 1996, MNRAS, 280, 67

Goldreich, P., \& Julian, W.H. 1969, ApJ, 157, 869

Hughes. P.A., Aller, H.D., \& Aller, M.F. 1985, ApJ, 298, 301

Illarionov, A.F., \& Krolik, J.H. 1996, ApJ, 469, 698

Kinman, T.D. 1977, Nature, 267, 798

Krichbaum, T.P. 1995, in:Quasars and AGN: High Resolution Radio Imaging, ed. M. Cohen and K. Kellerman, proceedings of a Conference of the National Academy of Sciences, Irvine, CA, USA

Levinson, A. 1998, ApJ, 507, 145, 1998

Livio, M., Ogilvie, G.I., \& Pringle, J.E. 1998, ApJ, in press

Lovelace, R.V.E. 1976, Nature, 262, 649

Lovelace, R.V.E., McAuslan, J., \& Burns, M. 1979, in Proceedings of La Jolla Institute Workshop on Particle Acceleration Mechanisms in Astrophysics, ed. J. Arons, C. Max, \& C. McKee (AIP, New York).

Lovelace, R.V.E., Newman, W.I., \& Romanova, M.M. 1997, ApJ 484, 628 (LNR97)

Lovelace, R.V.E., Romanova, M.M., \& Newman, W.I 1994, ApJ, 437, 136 (LRN94)

Lovelace, R.V.E., Wang, J.C.L., \& Sulkanen, M.E. 1987, ApJ, 315, 504

Lubow, S.H., Papaloizou, J.C.B., \& Pringle, J.E. 1994, MNRAS, 268, 1010

Macdonald, D.A., \& Thorn, K.S. 1982, MNRAS, 198, 345

Magdziarz, P., Moderski, R., \& Madejski, G.M. 1997, in: Relativistic Jets in $A G N s$, ed. M. Ostrowski, M. Sikora, G. Madejski and M. Begelman, Poland, Kraków, p.238

Mannheim, K., \& Biermann, P.L. 1992, A\&A, 253, L21

Maraschi, L., Ghisellini, G., \& Celotti, A. 1992, ApJ, 397, L5

Marscher, A.P 1980, ApJ, 235, 386

Marscher, A.P., \& Bloom, S.D. 1992, in The Compton Observatory Science Workshop, (ed. C.R.Shader, N. Gehrels, and B.Dennis) NASA CP-3137, 346

Mastichiadis, A., \& Kirk, J.G. 1997, A \& A, 320, 19 
Mattox, J.R., et al. 1997, ApJ, 476, 692

Pian, E., et al. 1998, ApJ, 492, L17

Protheroe, R.J. \& Biermann, P.L. 1997, APh, 6, 293

Punch, M. et al 1992, Nature 358, 477

Raga, A.C., Canto, J., Binette, L., \& Calvet, N. 1990, ApJ, 364, 601

Romanova, M.M., \& Lovelace, R.V.E. 1992, A\&A, 262, 26 (RL92)

Romanova, M.M., \& Lovelace, R.V.E. 1997, ApJ, 475, 97 (RL97)

Romanova, M.M., Ustyugova, G.V., Koldoba, A.V., Chechetkin, V.M., \& Lovelace R.V.E., 1997, ApJ, 482, 708

Romanova, M.M., Ustyugova, G.V., Koldoba, A.V., Chechetkin, V.M., \& Lovelace R.V.E., 1998, ApJ, 500, 703

Sambruna, R.M., Maraschi, L., \& Urry, C.M. 1996, ApJ, 463, 444

Sambruna, R.M., et al. 1997, ApJ, 474, 639

Sambruna, R.M., et al. 1998, ApJ, in press, astro-ph/9810319

Sikora, M., Begelman, M., \& Rees, M. 1994, ApJ, 421, 153

Takahara, F. 1997, in Relativistic Jets in AGNs, ed. M. Ostrowski, M. Sikora,

G. Madejski and M. Begelman, Poland, Kraków, p.170

Thompson, C. 1997, in Relativistic Jets in AGNs, ed. M. Ostrowski, M. Sikora,

G. Madejski and M. Begelman, Poland, Kraków, p.253

Wagner, S.J., \& Witzel, A. 1995, Ann. Rev. Astron. Astropys., 33, 163

Urry, M.C., \& Padovani, P. 1995, PASP, 107, 803

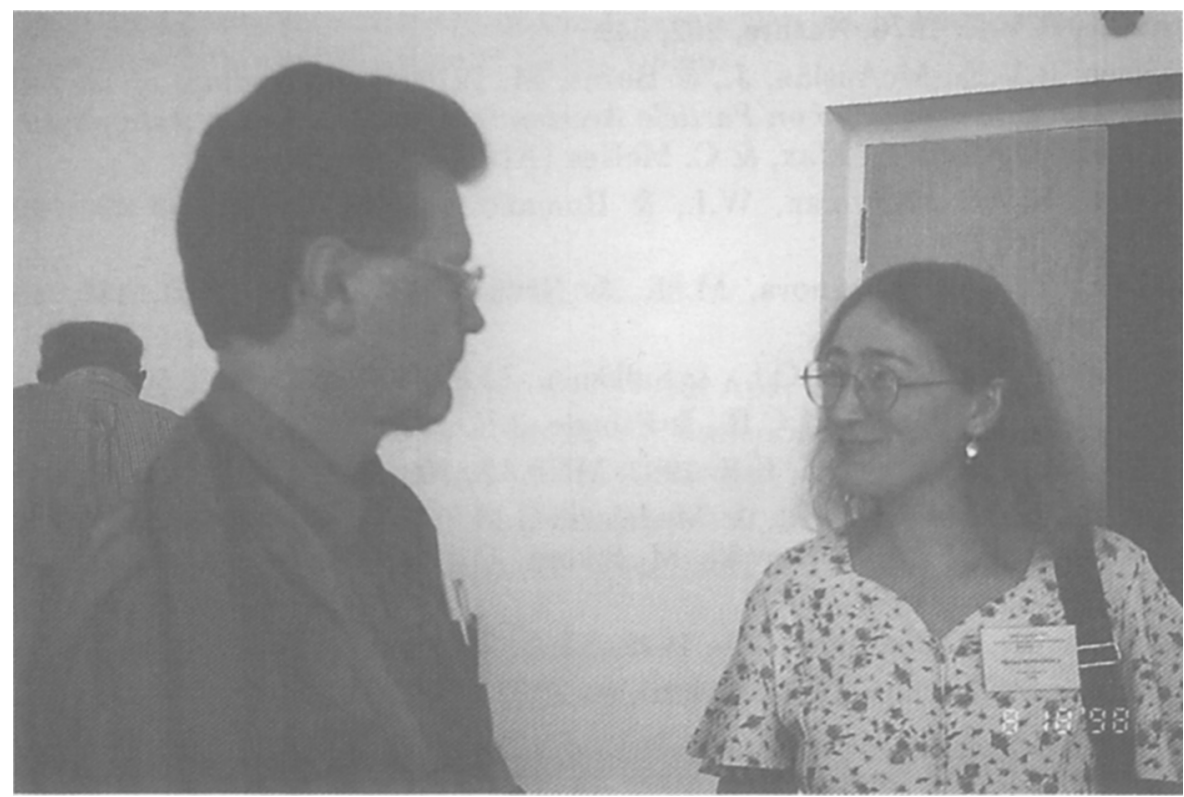

Dan Weedman and Marina Romanova 